\title{
Experimental Realisation of Single-carrier Alamouti-coded QPSK Using Frequency-Diverse Dual-Polarisation RF Pilot Tones
}

\author{
M. Sezer Erkılınç(1), Domaniç Lavery(1), Xu Zhou(2), Yansi Le ${ }^{(2)}$, Zhiyong Feng( $^{(2)}$, Polina Bayvel(1), \\ and Robert I. Killey ${ }^{(1)}$
}

(1) Optical Networks Group, Dept. of Electronic \& Electrical Engineering, University College London, Torrington Place, London, UK, WC1E 7JE, m.erkilinc@ee.ucl.ac.uk

(2) Networks Research Department, Huawei Technologies Co., Ltd., Shenzhen 518129, China

\begin{abstract}
A novel DSP chain using a pair of frequency-and polarisation-diverse RF pilot tones is proposed for single-carrier Alamouti-coded QPSK signal detection. The system's tolerance to static and dynamic signal polarisation is experimentally evaluated, with sensitivity penalties $\leq 1.5 \mathrm{~dB}$ demonstrated.
\end{abstract}

\section{Introduction}

The growth in residential and business user services will inevitably require higher bandwidth, and consequently, higher throughput optical access networks. Due to the limited receiver sensitivity of direct detection (DD) receivers, it becomes challenging to provide multi-gigabit (e.g., $\geq 5 \mathrm{~Gb} / \mathrm{s}$ ) per user, whilst maintaining similar power budgets $(\geq 30 \mathrm{~dB})$ for higher speed optical networks and preserving the passive optical network (PON) infrastructure already deployed. On the other hand, coherent technology underpinned by digital signal processing (DSP) exhibit significantly higher receiver sensitivities in comparison to DD receivers; a major advantage, particularly for future PON technologies ${ }^{1,2}$. Consequently, coherent transceivers offer higher power budgets, enabling higher split ratios (increased number of users), and longer reach (through the full compensation of linear channel impairments). This budget can be arbitrarily shared between reach and split ratio depending on the network requirements. Additionally, its wavelength selectivity enables colourless receiver operation, allowing the use of such receivers having identical design, based on tunable lasers, in an optical network unit (ONU); facilitating volume production and reducing inventory requirements.

In recent years, there has been a significant effort to reduce the complexity of a coherent receiver in order to make coherent technology viable for access networks. The optical complexity of a coherent receiver can be significantly reduced if polarisationindependent reception is realised, i.e., sacrifising polarisation-diversity. Low complexity coherent receiver designs, requiring only analogue processing (avoiding the need for DSP) and operating at 1.25 and $10 \mathrm{~Gb} / \mathrm{s}$ over, respectively, 60 and $44 \mathrm{~km}$ standard single mode fibre (SSMF) have been recently demonstrated ${ }^{3,4}$. While these schemes require neither analogue-to-digital converters (ADC) nor DSP to recover the signal, polarisation beam splitter/rotator is required in a receiver. On the other hand, polarisation scrambling applied in a transmitter has been proposed to avoid the requirement for polarisation tracking at the ONU receiver, and demonstrated in a system operating at $10 \mathrm{~Gb} / \mathrm{s}^{5}$. An alternative approach, using Alamouti (polarisation-time block) coding, which potentially offers better sensitivity, has recently attracted significant interest. Alamouti-coding with OFDM signalling technique, operating at 10.7 and $21.4 \mathrm{~Gb} / \mathrm{s}$, has been demonstrated ${ }^{6}$, whereas its operation with single-carrier signal formats has investigated to date only by means of simulations ${ }^{7,8}$.

In this paper, Alamouti-coded single-carrier operation, enabling coherent detection using no polarisation tracking unit in the receiver, is demonstrated experimentally for the first time. It leads to a significant simplification in the receiver design. Conventional DSP chain for typical single carrier transmission fails to recover the Alamouti-coded symbols since the orthogonality between the symbol pairs is lost due to the phase noise. Thus, here a novel DSP chain using a pair of frequency- and polarisation-diverse RF pilot tones is proposed, and its effectiveness is experimentally validated using a low-complexity coherent receiver operating at a net bit rate of $20 \mathrm{~Gb} / \mathrm{s}$. The performance of proposed signalling scheme is assessed, and shown to be robust, for both static and dynamically varying polarisation states.

\section{Experimental configuration}

The experimental configuration is shown in Fig. 1. An external cavity laser (ECL), operating at $1550 \mathrm{~nm}$ (100 kHz linewidth) with $13 \mathrm{dBm}$ output power, was used as an optical source for the integrated dualpolarization (DP) IQ-modulator. Four electrical signals, generated using four $90 \mathrm{GSa} / \mathrm{s}$ Keysight digitalto-analogue (DAC) converters, were used to drive the DP IQ-modulator. To evaluate the resilience of 10.7 GBaud Alamouti-coded QPSK signal to different signal polarization states (i.e., emulating static polar- 


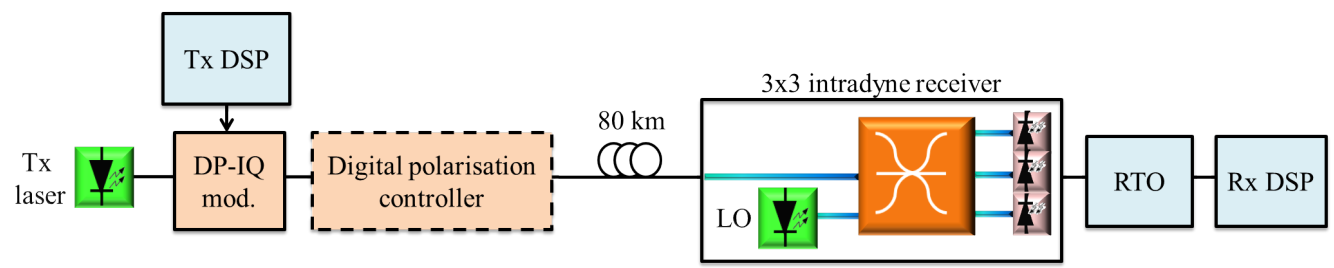

Fig. 1: Experimental configuration to realise single carrier Alamouti-coded QPSK signal. RTO: Real-time oscilloscope

isation rotation), a digitally operated polarization controller (DPC) was used to rotate the signal over the full Poincare sphere. Moreover, to emulate dynamic polarisation rotation, the generated waveforms were pre-rotated digitally at a range of polarisation rotation frequencies to assess the tolerance of the system to dynamically varying polarisation states. Finally, the signal was transmitted over $80 \mathrm{~km}$ of SSMF with an attenuation of $15.2 \mathrm{~dB}(0.19 \mathrm{~dB} / \mathrm{km})$ and a chromatic dispersion coefficient of $16.8 \mathrm{ps} / \mathrm{nm} / \mathrm{km}$ at $1550 \mathrm{~nm}$, to demonstrate a long-reach optical access link.

The transmitted signal was detected using a low complexity intradyne coherent receiver consisting of a $3 \times 3$ coupler followed by three single-ended PIN photodiodes (PDs). A second ECL was used as the local oscillator, emitting light approximately $\sim 300 \mathrm{MHz}$ away from the transmitter laser. The received signal was digitised using an $80 \mathrm{GSa} / \mathrm{s}$ $33 \mathrm{GHz}$ bandwidth real-time oscilloscope.

\section{Experimental configuration}

In the transmitter DSP, the Nyquist-shaped Alamouticoded dual-polarisation QPSK (DP-QPSK) waveforms were generated using a pair of frequencydiverse RF pilot tones, each inserted on an orthogonal polarisation state. Following bit-to-symbol mapping, the Alamouti coding ${ }^{9}$ was applied, as shown in Fig. 2(a). A Nyquist root raised cosine filter with a roll-off factor of 0.01 was applied. To avoid power fading in the presence of polarisation rotation, the RF pilot tones were inserted at a relative frequency $\omega_{R F}$ of $5.85 \mathrm{GHz}$ on ( $500 \mathrm{MHz}$ away from) both sides of the spectrum, as shown in the inset of Fig. 2. The pilot tone-to-signal power ratio was set to $-8 \mathrm{~dB}$, as further discussed in the next section.
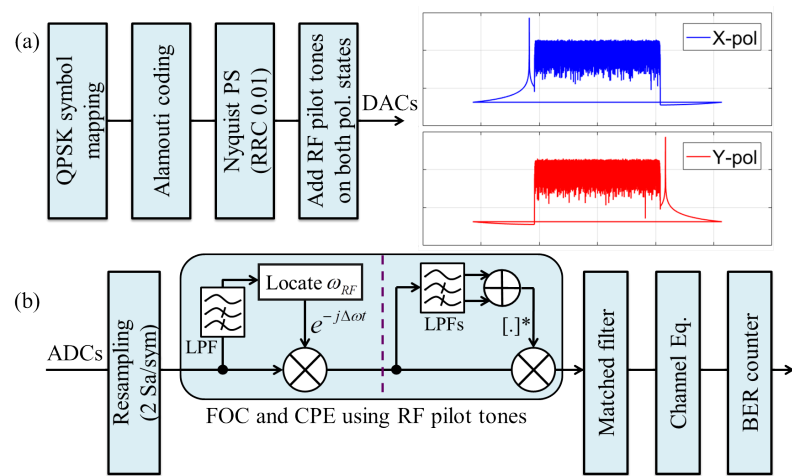

Fig. 2: DSP performed at the (a) transmitter and (b) receiver. $\Delta \omega: \omega_{I F}-\omega_{R F}$. PS: Pulse shaping
In the receiver DSP, the signal was resampled to 2 samples/symbol and normalised to unit power. Using the RF pilot tones, frequency offset correction (FOC) and carrier phase recovery (CPR) were achieved. For FOC, $3^{\text {rd }}$-order Gaussian-shaped low pass filters (LPFs) with $1 \mathrm{GHz}$ bandwidth was applied, and the relative intermediate frequency of pilot tones $\omega_{I F}$ were located using peak search. The FO was estimated by subtracting $\omega_{R F}$ from $\omega_{I F}$, and corrected using one of the pilot tones since its $\omega_{R F}$ was known a-priori. Subsequently, two $5^{\text {th }}$-order Butterworth LPFs was used for phase recovery. The two low-pass filtered RF pilot tones distorted by the system phase noise were first averaged, conjugated and multiplied with the received signal to mitigate the phase noise, as shown in Fig. 2(b). Note that the LPFs' bandwidth depends on the system phase noise and signal-to-noise ratio (SNR), and hence, was optimised to maximise the performance, e.g., set to $20 \mathrm{MHz}$ bandwidth at $-42 \mathrm{dBm}$ received power. Following this stage, a matched filter was applied and channel equalisation was performed using an Alamouti equaliser, explained in ${ }^{7}$ but requiring no phase recovery. Finally, the errors after symbol-to-bit mapping were counted using $2^{19}$ symbols.

\section{Results and Discussion}

Initially, at a fixed signal power of $-41 \mathrm{dBm}$, the RF pilot tone power was varied to obtain the optimum RF pilot tone-to-signal power ratio for carrier phase recovery. This stage is crucial to successfully recover the transmitted symbols. The optimum RF pilot toneto-signal power ratio was found to be $-8 \mathrm{~dB}$, as shown in Fig. 3. At lower values, the pilot tone is not sufficiently above the noise level to obtain accurate phase information, whereas SNR degrades when the pilot tone power is too high.

The system performance was then measured over 625 equally-spaced static polarization states over the Poincare sphere. The measurements were taken at $-43 \mathrm{dBm}$ received signal power. The BER distribution is plotted in Fig. 4. The sensitivity penalty was found to be within $1.5 \mathrm{~dB}$, i.e., requiring additional $\leq 1.5 \mathrm{~dB}$ to achieve BER values below the HD-FEC threshold, assumed to be $4 \times 10^{-3}$, for the signals exhibiting BERs above the HD-FEC threshold, plotted in Fig. 4. Following this, the receiver was tested with dy- 


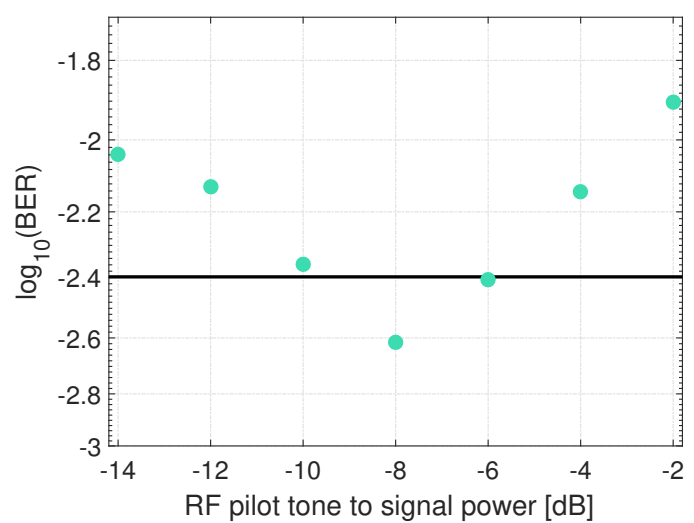

Fig. 3: RF pilot tone-to-signal power ratio used at carrier phase

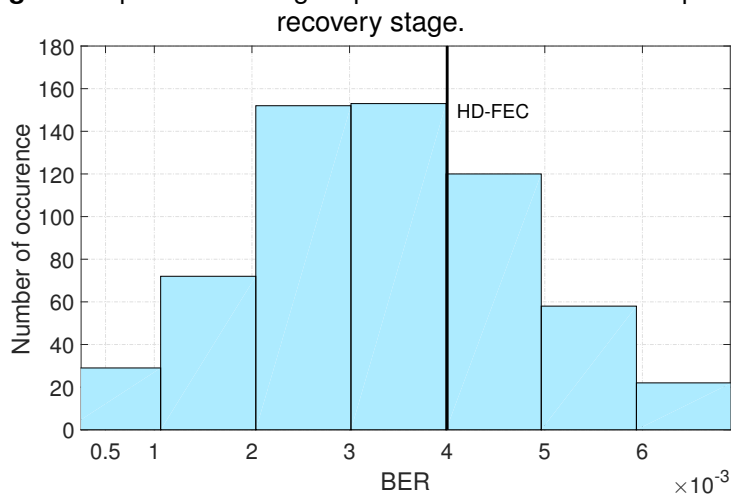

Fig. 4: The number of occurrence for 625 equally-spaced static polarization states versus BER.

namic polarisation rotation over a range of rotation frequencies. It was found that a sensitivity penalty of only $0.5 \mathrm{~dB}$ occurred for frequencies of up to $100 \mathrm{kHz}$ and increasing to $3.5 \mathrm{~dB}$ at a frequency of $500 \mathrm{kHz}$, as shown in Fig. 5 since the Alamouti equaliser failed to track the polarisation rotations at such frequencies. Nevertheless, the penalty up to $100 \mathrm{kHz}$ is acceptable, considering that typical polarisation rotation in installed fibres is at $\sim 15 \mathrm{kHz}$.

Finally, the sensitivity performance of the simplified coherent receiver was investigated in back-to-back operation and transmission over $80 \mathrm{~km}$ of SSMF. It is important to note that a polarisation controller was inserted before the receiver to ensure that the system operated in the worst-case scenario, that is with a $\pm 45^{\circ}$ polarization rotation between the two polarisation states of the signal and that of the local oscillator. The receiver BER versus received power are shown in Fig. 6 . The receiver sensitivity at the HD-FEC limit was found to be $-42 \mathrm{dBm}$, and negligible sensitivity difference was observed in transmission case.

\section{Conclusions}

The Alamouti-coded single-carrier QPSK signal was experimentally demonstrated using a pair of frequency- and polarisation-diverse RF pilot tones. The system performance was assessed for static and dynamically varying polarisation states. Sensitivity penalties of $\leq 1.5 \mathrm{~dB}$ were observed for polarisation rotation frequencies of up to $100 \mathrm{kHz}$ using the pro-

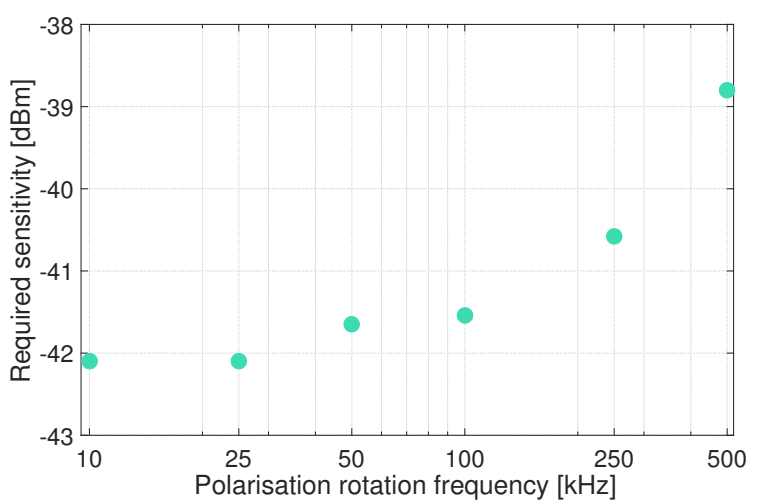

Fig. 5: The receiver sensitivity at the HD-FEC limit with respect

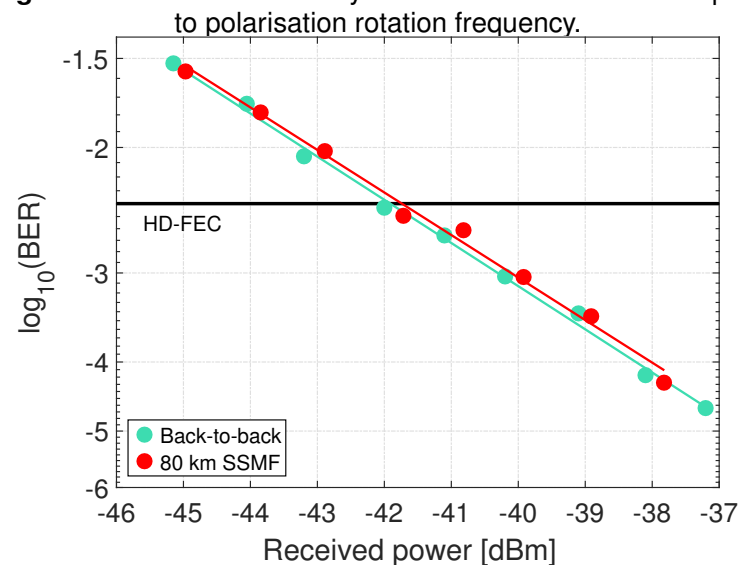

Fig. 6: The receiver sensitivity performance of 10.7 GBaud Alamouti-coded QPSK signal.

posed novel DSP chain.

\section{References}

[1] H. Rohde et al., "Coherent ultra dense WDM technology for next generation optical metro and access networks," J. Lightw. Technol., vol. 32, no. 10, pp. 2041-2052, 2014.

[2] A. Shahpari et al., "Coherent access: A review," J. of Lightw. Technol., vol. 35, no. 4, pp. 1050-1058 (2017).

[3] J.A. Altabas et al., "Real-time $10 \mathrm{Gbps}$ polarization independent quasicoherent receiver for NG-PON2 access networks," Proc. OFC, Th1A.3 (2018).

[4] F. Bottoni et al., "Coherent PON system with high-sensitivity polarization-independent receiver and no ADC/DSP," Proc. ECOC (2015).

[5] I.N. Cano et al., "Polarization independent single-PD coherent ONU receiver with centralized scrambling in udWDM-PONs," Proc. ECOC, P.7.12 (2014).

[6] M.S. Erkılınç et al., "Bidirectional wavelength-division multiplexing transmission over installed fibre using a simplified optical coherent access transceiver," Nature Commun., vol. 8, no. 1043 (2017).

[7] Md.S. Faruk et al., "DSP algorithms for recovering singlecarrier Alamouti coded signals for PON applications," Opt. Express, vol. 24, no. 21, pp. 24083-24091 (2016).

[8] Md.S. Faruk et al., "Technology Requirements for an Alamouticoded $100 \mathrm{~Gb} / \mathrm{s}$ digital coherent receiver using $3 \times 3$ couplers for passive optical networks," vol. 10, no. 1 (2018).

[9] M.S. Erkılınç et al., "Polarization-insensitive single-balanced photodiode coherent receiver for long-reach WDM-PONs," J. Lightw. Technol., vol. 34, no. 8, pp. 2034-2041 (2016). 